\title{
Elemente und Ephemeride des Planeten (116). *)
}

Von der Güte des Herrn Admiral Sands eine Washingtoner Beobachtung vom 16. September erhaltend, legte ich dic folgenden drei Orte einer ersten Babnbestimnung zu Grunde: 1871

$\overbrace{}^{1871}$

Sept.8

$0414=6^{\prime} 51-3^{\circ}+4^{\prime} 38^{\prime \prime} 0$

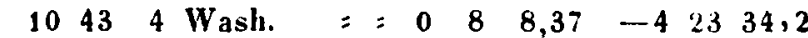

$=24121555$ Ham.Coll. $==\begin{array}{lllll}0 & 1 & 36,30 & -5 & 3\end{array} 4,1$ und erhielt danit die folgenden Elemente:

Epoche: 1871 Jan. 0 m. Zt. Berlin.

$$
\begin{aligned}
M_{\mathrm{o}} & =162^{\circ} 5^{\prime} 34^{\prime \prime} 4 \\
\pi & =1465811,4 \\
\Omega & =6547 \\
i & =32926,9 \\
\varphi & =54514,05 \\
\mu & =765^{\prime \prime} 503 \\
\log a & =0,4440398
\end{aligned}
$$

nebst den Coordinatengleichungen, (auf dasselbe mittlere Aequinoctium bezogen):

$$
\begin{aligned}
& x=r(9,9993295) \sin \left[236^{\circ} 55^{\prime} 48^{\prime \prime} 1+v\right] \\
& y=r(9,9578104) \sin [1482420,7+v] \\
& z=r(9,6272312) \sin [140618,6+v]
\end{aligned}
$$

\begin{tabular}{|c|c|c|c|}
\hline $0^{h}$ Berl. m. Zt. & $\alpha$ (116) & $d 110$ & $\log \Delta$ \\
\hline 1871 Oct. 15 & $23^{h} 46^{m} 22^{s}$ & $-6^{\circ} 21^{\prime} 3$ & 0,3172 \\
\hline 16 & $45 \quad 45$ & 24,0 & 0,3185 \\
\hline 17 & 458 & 26,5 & 0,3198 \\
\hline 18 & $44 \quad 33$ & 28,8 & 0,3212 \\
\hline 19 & 4358 & 31,0 & 0,3226 \\
\hline 20 & 4325 & 33,1 & $0,32+1$ \\
\hline 21 & 4253 & 35,0 & 0,3256 \\
\hline 22 & 4222 & 36,8 & 0,3272 \\
\hline 23 & 4152 & 38,4 & 0,3288 \\
\hline 24 & 4123 & 39,9 & 0,3304 \\
\hline 25 & 4056 & 41,2 & 0,3321 \\
\hline 26 & $40 \quad 29$ & 42,4 & 0,3338 \\
\hline 27 & 404 & 43,4 & 0,3356 \\
\hline 28 & 3940 & 44,3 & 0,3374 \\
\hline 29 & $\begin{array}{ll}39 & 18\end{array}$ & 45,0 & 0,3392 \\
\hline 30 & 3858 & 45,6 & 0,3411 \\
\hline 31 & $\begin{array}{lll}23 & 38 & 39\end{array}$ & $-646,0$ & 0,3430 \\
\hline
\end{tabular}

Die folgende Beobachtung:

$\frac{1871}{\text { Oct. } 2} \underbrace{\text { Ham. Coll.m. Zt. }}_{10^{\mathrm{h}} 23^{\mathrm{m}} 50^{\mathrm{s}}}$

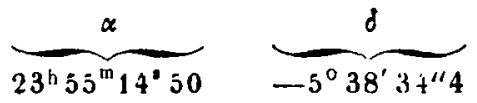

- wenn man die Rectascension desVergleichsternes in Weisse's Catalog $23^{h}$, $1099 \mathrm{um}-1^{\text {s }}$ corrigirt, wie es sein muss, zufolge Vergleichungen mit mehreren benachbarten Sternen, wird durch obige Elemente fast genau wiedergegeben. Dio bier angelügte Ephemeride wird daher den Planeten, der hell 11 ter Grösse ist, sicher auffinden lassen.

*) In TE $186 t$ und 1862 and die Nummern der beiden neuesten Planeten vertauscht; dic richtige Reihenfolge ist: 116, entdeckt von C. II. F. Peters 1871 September 8.

117) Lomia, cutdecht von Borelly 1871 September 12.

\section{Beobachtung des Encke'schen Cometen.}

Whatever the object may have been which we olserved here on Sept. 22 (and I shall examine the matter furlher on the first really good night) it appears certain that it was not Encke's comet, as I had first supposed.

I found the comet on Oct. 8 as a large, faint and very diffused nebulosity, and secured an approxinate position giving for the correction of the ephemeris, $-39^{5}$ in $R$. A. and $-8^{\prime} 6$ in Decl.

Last night I obtained a much better place ly comparison with Argelauder Zoue $+36^{\prime \prime}$, No 241 , with the sanis barnicrometer - "Micrometer von scbmalen Blechsfreife" that was employed at Mr. Bishop's Observatory by my late friend Dr. E. Vogel, in his first observation of kinckes comet 1852 Jan. 9 (Astr. Nachr. N8 792).
The result is

Oct. 10 at $10^{\text {h }} 48^{\text {in }} 48^{\text {s }}$ Tivickenham M. T.

$$
\text { R. A. }=18^{\circ} 51^{\prime} 34^{\prime \prime}
$$

Decl. $=+36$ is 47

giving for the correction of the ephemeris

$$
\begin{aligned}
& \text { R. A. }=-36^{\prime} 5 \\
& \text { Decl. }=-9^{\prime} 11^{\prime \prime}
\end{aligned}
$$

The state of the weather here in the absence of moonlight prevented any search for the comet between Sept. 22 aud Oct. 8.

The comet is an exceedingly difficult object to olsserve, from its very diflused appearance.

Twickenham, 1871 0ct. 11 J. R. Hind.

\section{Beobachtung des Encke'schen Cometen.}

Der Conet wurde hier zuerst den 4. October um 8 Uhr von Dr. Dunér gefunden. Er erschien als ein sehr schwacher Nebel, welcher $27^{5}$ nach dem Sterne Lal. 3089 folgte und etwa $8^{\prime}$ südlicher stand. Den 5 . October $7^{\text {h }} 30^{\mathrm{m}}$ sah ich den Nebel wieder; er ging dann demselhen Sterwe $2^{1 n} 7^{5}$ voran und war $8^{\prime}$ nördlicher. An beiden Tagen war es jedoch unmöglich den Cometen ordentlich zu beobachten; erst gestern gelang mir folgende Bestimnung, welche ich bis auf $\pm 15^{\prime \prime}$ sicher schätze:

$$
\begin{aligned}
& 1871 \text { Oct. } 10 \\
& \text { Mittl. Zt. Lund. } \\
& 10^{\mathrm{h}} 49^{\mathrm{gl}} 32^{\mathrm{s}} \\
& \frac{\text { AR } \text { ó }^{\prime}}{1^{\mathrm{h}} 15^{\mathrm{n}} 31^{\circ} 85} \\
& \frac{\text { Decl. } \sigma^{\prime}}{+36^{\circ} 15^{\prime} 1^{\prime \prime} 8} \\
& \text { Vergleiclustern }=\text { Weisse } 1^{\mathrm{h}}, 298 \text {. }
\end{aligned}
$$

Corr. der Eph.

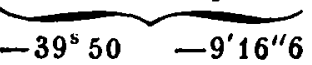

